\title{
Erstes Symposium junger Strafrechtswissenschaftlerinnen und Strafrechtler in Köln
}

Johannes Kaspar*

Aufbruchsstimmung in Köln: Die Privatdozenten Dr. Brian Valerius (Würzburg) und Dr. Andreas Popp (Passau) sowie Prof. Dr. Georg Steinberg (Köln) hatten am 19. und 20.11.2010 zum „Symposium junger Strafrechtlerinnen und Strafrechtler“ geladen - und stießen damit auf große Resonanz. Über 50 Teilnehmer, durchweg promoviert und ganz überwiegend als Habilitandinnen und Habilitanden an Lehrstühlen in ganz Deutschland beschäftigt, folgten dem Ruf in die Domstadt und diskutierten über „Das Wirtschaftsstrafrecht des StGB in der aktuellen höchstrichterlichen Rechtsprechung“, das Gegenstand mehrerer Vorträge war. Damit war soweit ersichtlich zum ersten Mal ein beträchtlicher Anteil des deutschen strafrechtswissenschaftlichen Nachwuchses auf einer eigenen Tagung versammelt. Ein voller Erfolg, der aber nicht ganz überraschend kam: zu groß, zu offensichtlich war der Bedarf nach einem solchen Forum, in dessen Rahmen sich junge Strafrechtswissenschaftlerinnen und -wissenschaftler zum zwanglosen persönlichen und fachlichen Austausch treffen können. Gerade die Möglichkeit, wissenschaftliche Vorträge im Kollegenkreis zu halten und über ihren Inhalt zu diskutieren, bestand für diese Personengruppe bislang nicht. Mit der äußerst verdienstvollen Initiative von Valerius, Popp und Steinberg wurde diese veritable „Marktlücke“ nun geschlossen.

Als neben dem fachlichen Ertrag sicher wichtigstes Ergebnis kann festgehalten werden, dass die Tagung nach dem Willen der Teilnehmer in Zukunft regelmäßig statt finden soll. Wie die vergleichbaren Veranstaltungen der Kollegen aus dem öffentlichrechtlichen und zivilrechtlichen Bereich sollen die Tagungen abwechselnd in verschiedenen Städten ausgerichtet werden, wobei sich erfreulicherweise für die nächsten drei Male bereits Interessenten gemeldet haben. Das nächste Treffen ist bereits in Planung und wird voraussichtlich im Frühjahr 2012 in Berlin stattfinden, organisiert von Habilitandinnen und Habilitanden der Humboldt-Universität, die dabei von Kollegen der LMU München unterstützt werden. Auch weitere wichtige Weichenstellungen wurden auf der Kölner Gründungssitzung beschlossen. So soll in der Folgezeit nach dem Willen der Mehrzahl der Teilnehmer ein jährlicher Turnus angestrebt werden, um einen regelmäßigen Kontakt während der zeitlich nur begrenzten Habilitationsphase sicher zu stellen. Weiterhin soll die Tagung nicht auf Habilitierende beschränkt, sondern auch für sonstige Interessierte einschließlich Studierende und Doktoranden geöffnet werden. Die Beiträge der zukünftigen Veranstaltungen sollen (wie bereits die Kölner Vorträge) in einem vom Nomos-Verlag herausgegebenen Tagungsband veröffentlicht werden. Und um eine gewisse organisa-

* Dr. Johannes Kaspar ist Habilitand am Institut für die Gesamten Strafrechtswissenschaften der LudwigMaximilians-Universität München. 
torische Struktur und Kontinuität sicher zu stellen, wurden mit Dr. Beatrice Brunhöber (Berlin) und Dr. Nina Nestler (Würzburg) zwei dauerhafte Ansprechpartnerinnen gewählt, die die weiteren Planungen koordinieren sollen. Die Einrichtung einer Homepage ist in Vorbereitung.

Diesen abschließenden Entscheidungen waren zwei interessante und ertragreiche Tage vorausgegangen. Am Mittag des 19.11.2010 hatten sich die Teilnehmer in den Räumen des Juristischen Seminars der Universität eingefunden und wurden zunächst von Prof. Dr. Thomas Weigend in seiner Eigenschaft als Dekan der juristischen Fakultät sowie von Prof. Dr. Claus Kreß, dem Geschäftsführenden Direktor des Instituts für Strafrecht und Strafprozessrecht, freundlich empfangen. Beide zeigten sich in ihren Grußworten sehr erfreut, dass gerade Köln als Austragungsort des Symposiums gewählt worden war und wiesen auf die lange Tradition der rechtswissenschaftlichen Ausbildung an der Kölner Universität sowie den Stellenwert des Strafrechts innerhalb der Fakultät hin.

An den folgenden zwei Tagen wurden in insgesamt acht Vorträgen aktuelle höchstrichterliche Entscheidungen aus dem Bereich des Wirtschaftsstrafrechts beleuchtet und in offener, durchaus kritischer, aber stets sachlicher und angenehmer Atmosphäre diskutiert.

Den Anfang machte Dr. Bettina Noltenius (Bonn), die über „Betrug kraft Organisationsherrschaft (mittelbare Täterschaft) in der neueren höchstrichterlichen Rechtsprechung " sprach. Sie verwies einleitend auf die Entscheidung des Bundesgerichtshofs zur Strafbarkeit ehemaliger DDR-Funktionäre, die als „Täter hinter dem Täter“ aufgrund ihrer objektiven Tatherrschaft für Taten ihrer Untergebenen verurteilt worden waren. In einem obiter dictum war damals auf die Möglichkeit der Anwendung dieser Grundsätze auch auf die Organisationsstruktur von Unternehmen hingewiesen worden. In neueren Entscheidungen (u. a. BGHSt 48, 331) wurde dieser Gedanke nun aufgegriffen und eine Betrugstäterschaft von Führungskräften in Unternehmen kraft Organisationsherrschaft angenommen. Noltenius kritisierte, dass die Anforderungen an die Täterschaft zu weit abgesenkt würden, wenn man mit dem BGH in objektiver Hinsicht die bloße Möglichkeit einer Lenkung des unmittelbar handelnden Vordermanns durch die hierarchische Organisationsstruktur sowie auf subjektiver Ebene allein einen generellen Willen zur Tatherrschaft ohne Vorsatz bezüglich konkreter Täuschungshandlungen genügen lasse. In der anschließenden Diskussion wurde zudem auf die von Roxin formulierte Einschränkung hingewiesen, dass eine mittelbare Täterschaft kraft Organisationsherrschaft bei Wirtschaftsunternehmen allenfalls bei Annahme genereller „Losgelöstheit vom Recht“ angenommen werden könne. 
Privatdozentin Bettina Weißer (Köln) widmete sich im Anschluss der Frage der „Wissenszurechnung im arbeitsteiligen Unternehmen“, die sich innerhalb des Betrugstatbestandes am erforderlichen Irrtum des Opfers festmachen lässt. War es im vorangegangenen Vortrag noch um die Probleme gegangen, die durch Mehrpersonenverhältnisse auf Täterseite entstehen, wurde der Blick somit nun auf die Auswirkung arbeitsteiliger Strukturen auf der Seite der Geschädigten gelenkt. Bei Irrtum eines verfügenden Entscheidungsträgers wurde zunächst überzeugend dargelegt, dass besseres Wissen von untergeordneten Unternehmensangehörigen oder Hilfspersonen nichts an der Erfüllung des Betrugstatbestandes ändern könne; der Rechtsgedanke von $\$ 166$ BGB könne hier nicht zu einer „Wissenszurechnung“ führen, die den Irrtum ausschließen könnte. Wie aber ist der Fall zu entscheiden, in dem Mitarbeiter bei der ihnen obliegenden Vermögensverfügung zwar einem täuschungsbedingten Irrtum unterliegen, vorgesetzte Unternehmensangehörige auf höheren Hierarchieebenen die tatsächliche Sachlage aber kennen? Der BGH (etwa in BGH NStZ-RR 2010, 146) tendiert hier stark zur Ablehnung einer täuschungs- und irrtumsbedingten Verfügung. Weißer plädierte demgegenüber für eine Differenzierung nach der Stellung des bösgläubigen Vertreters auf Opferseite: eine Wissenszurechnung sei nur zu bejahen, wenn Personen auf höchster Hierarchieebene, die quasi als „Repräsentanten des Vermögens“ fungierten, über die Täuschung Bescheid wüssten. Hier sei von einer eigenverantwortlichen Selbstschädigung auszugehen und daher eine Betrugsstrafbarkeit abzulehnen. Dies rief allerdings Kritik hervor: es wurde eingewandt, dass man zwischen dem Vermögensinhaber und dessen Vertreter (selbst auf höchster Ebene) unterscheiden müsse, im Falle einer GmbH also zwischen den Gesellschaftern als Trägern des Vermögens einerseits und dem Geschäftsführer als Vertreter der GmbH. Wenn letzterer dem Betrugsgeschehen pflichtwidrig seinen Lauf lasse, sei nicht einzusehen, warum dies automatisch den Gesellschaftern zugerechnet werden solle.

Ein Thema, das derzeit in aller Munde ist, wurde sodann von Dr. Bijan Fateh-Moghadam (Münster) aufgegriffen: er sprach über „Criminal Compliance ernstgenommen - zur strafrechtlichen Garantenstellung des Compliance-Beauftragten “. Er wies zunächst (mit grafischer Untermalung) auf die Blitzkarriere des Begriffs der „Compliance“ hin. Es gehe dabei um den Schutz des Unternehmens vor finanziellen Nachteilen durch Straftaten, die aus dem Unternehmen heraus begangen werden; zentrale Funktion der Compliance sei damit letztlich die Senkung von Haftungsrisiken für die Unternehmen. Nun habe der Bundesgerichtshof in einem obiter dictum in seiner Entscheidung zu den Berliner Stadtwerken (BGHSt 54, 44) die Ansicht geäußert, wonach ein Compliance-Beauftragter regelmäßig Garant im Sinne von $\$ 13$ StGB sei. Dies folge als „notwendige Kehrseite“ aus der gegenüber der Unternehmensleitung übernommenen Pflicht zur Verhinderung von Rechtsverstößen. Nach Fateh-Moghadam sei eine Überwachungsgarantenstellung unter zwei Voraussetzungen denk- 
bar: zum einen müsse eine „Geschäftsherrenhaftung “ der Unternehmensleitung für Straftaten von Unternehmensangehörigen bestehen, zum zweiten müsse diese Verantwortlichkeit durch Delegation und tatsächliche Übernahme auf den ComplianceBeauftragten übertragen worden sein. Beides wurde von Fateh-Moghadam für möglich gehalten: eine Geschäftsherrenhaftung aufgrund einer Herrschaft über betriebsspezifische Gefahren sei zu bejahen und könne grundsätzlich auf den ComplianceBeauftragten ausgedehnt (nicht: abgewälzt) werden. Die Einrichtung einer Compliance-Organisation sei letztlich ambivalent: sie könne zwar zu einer gewissen Entlastung der Unternehmensspitze bei der Frage fahrlässiger Aufsichtspflichtverletzung führen, lasse deren Geschäftsherrenhaftung im Übrigen aber unberührt und begründe eine zusätzliche strafrechtliche Haftung für den Beauftragten. Abschließend wurde vom Referenten noch aus systemtheoretischer Perspektive bezweifelt, dass das Strafrecht unmittelbar „die Wirtschaft“ als eigenständiges System beeinflussen und steuern könne -hier sei allenfalls eine „Irritation“ möglich (und, wie man hinzufügen kann: eine solche dürfte die BGH-Entscheidung nach allem, was man hört, in Unternehmenskreisen in der Tat ausgelöst haben).

Privatdozent Dr. Brian Valerius (Würzburg) referierte anhand von mehreren Entscheidungen des BGH (u. a. BGHSt 53, 199) über die Problematik des Vermögensschadens bei Risikogeschäften. In einem Fall von Anlagebetrug hatte die Broschüre zwar Hinweise auf Risiken enthalten; diese seien aber heruntergespielt und zugleich eine Rendite in Höhe von 30 \% in Aussicht gestellt worden. Eine andere Entscheidung betraf ein sogenanntes Schneeballsystem, bei dem von den insgesamt von den Anlegern eingezahlten Beträgen in Höhe von 28 Mio. Euro nur 7 Mio. Euro ausgeschüttet worden waren. Bei der Bestimmung des Vermögensschadens, so Valerius, müsse nach allgemeinen Maßstäben eine Gesamtsaldierung erfolgen, bei der Leistung und Gegenleistung verglichen werden müssten. Dies bestimme sich anhand der jeweiligen Gewinn- und Verlustrisiken; nicht etwa dürfe allein von der Kombination aus Täuschung und Verlustrisiko auf einen Schaden geschlossen werden. Führe die Saldierung zu keinem objektiven Schaden, sei eine Schadensbegründung auch wegen eines individuellen Schadenseinschlags denkbar, hier aufgrund individueller Nutzlosigkeit der Leistung, wenn die Anlageform aufgrund großer objektiver Risiken nicht den Wünschen des risikoscheuen Anlegers entspreche.

Am Ende des ersten Tages sprach Dr. Till Zimmermann (Passau) über „Untreue durch Kreditvergabe" und bezog sich dabei auf eine neuere Entscheidung des BGH (wistra 2010, 21). Der Vorstand eines Kreditinstituts bewege sich in einem Spannungsfeld: er habe einerseits die Pflicht, mit dessen Vermögen sorgfältig umzugehen, andererseits sei für ein erfolgreiches Wirtschaften das Eingehen von Risiken unumgänglich. Bei der Vergabe von Krediten habe eine sorgfältige Prüfung der Vergabevoraussetzungen zu erfolgen, wozu auch eine Beschaffung von Informationen über 
den Kreditnehmer aus erster Hand gehöre. Im Hinblick auf den Untreuevorsatz genügt nach Zimmermann auch bei Annahme einer Vermögensgefährdung bloßer dolus eventualis hinsichtlich eben dieser Gefährdung, ein darüber hinaus gehender Vorsatz bezüglich der Realisierung des Risikos sei mit dem 3. Senat und entgegen der Auffassung anderer Senate nicht erforderlich. Daher genüge bei der Vergabe riskanter Kredite, dass der Täter mangels ausreichender Informationsbeschaffung mit Umständen rechne, die ein erhöhtes Risiko des Ausfalls des Kredits und damit einen Gefährdungsschaden begründen.

Den zweiten Tag eröffnete Privatdozent Dr. Andreas Popp (Passau). Er sprach über „Pflichtenakzessorietät und Irrtumslehre am Beispiel des $\$ 266$ a StGB “. Er erläuterte die Problematik anhand eines vom BGH (NStZ 2010, 337) kürzlich entschiedenen Falles, bei dem 90 polnische Staatsangehörige als Prospektverteiler beschäftigt waren und nach den faktischen Verhältnissen als Arbeitnehmer, der Angeklagte umgekehrt als Arbeitgeber im Sinne des Sozialversicherungsrechts zu qualifizieren war. Durch das Unterlassen der Zahlung von Sozialversicherungsbeiträgen habe sich der Angeklagte nach Ansicht des BGH gem. $\$ 266$ a StGB strafbar gemacht. Für seinen Tatbestandsvorsatz, so der BGH, genüge die Kenntnis der tatsächlichen Umstände, die seine Arbeitgebereigenschaft begründen; eine mögliche Fehlvorstellung über die damit verbundenen Pflichten sei lediglich ein (im konkreten Fall vermeidbarer) Verbotsirrtum gem. $\mathbb{1} 17$ StGB. Das wurde von Popp in Frage gestellt; die Entscheidung missachte, dass die Arbeitgeberstellung eine zweifache Bedeutung habe: einerseits sei damit die Position als Schuldner der sozialversicherungsrechtlichen Zahlungspflicht verbunden, andererseits diejenige des Adressaten des strafbewehrten Handlungsgebots und damit des potenziellen Unterlassungstäters. Beide Positionen fielen auch nicht notwendigerweise zusammen: so seien bei einem Unternehmen die juristische Person Schuldnerin der Zahlungspflicht, aber die verantwortlichen natürlichen Personen innerhalb des Unternehmens die Adressaten der Norm des $\$ 266$ a StGB. Für eine Erfüllung des Tatbestandsvorsatzes sei daher mehr zu fordern als die bloße Kenntnis der den Arbeitgeberstatus begründenden Umstände. Vielmehr müsse der erforderliche soziale Sinn der pönalisierten Nicht-Zahlung erfasst werden; zu verlangen sei also eine Art „Bedeutungskenntnis“ im Hinblick auf die mit dem Arbeitgeberstatus verbundenen Pflichten bzw. die Kenntnis der „tatbestandsmäßigen Situation“. Das Anliegen der Rechtsprechung, Schutzbehauptungen der Täter entgegen zu treten, müsse ggf. auf anderem Wege verfolgt werden, etwa durch eine (offen gelegte) Normativierung des Vorsatzbegriffs oder durch eine Absenkung der prozessualen Anforderungen an den Vorsatznachweis.

Der folgende Vortrag von Dr. Andrea Hagemeier (Heidelberg) befasste sich mit einer aktuellen Entscheidung des BGH (NStZ 2009, 635) zum Tatbestand des Bankrotts. Konkret ging es um die Strafbarkeit einer sogenannten „Firmenbestattung “ einer an 
sich schon zahlungsunfähigen $\mathrm{GmbH}$, bei der der Angeklagte die Gesellschaftsanteile sowie die Geschäftsführung auf Strohmänner übertrug, sich aber umfassende Verfügungsmöglichkeiten sowie eine Rückkaufoption erhielt. Dazu kam eine Sitzverlegung und Umfirmierung. Der BGH ging ohne endgültige Festlegung davon aus, dass dies unter $\$ 283$ I Nr. 8 StGB bzw. "gegebenenfalls vorrangig“ unter Nr. 4 subsumiert werden könne. Letzteres setzt allerdings ein „Vortäuschen von Rechten anderer“ oder die „Anerkennung erdichteter Rechte“ voraus, was in Bezug auf den Verkauf der GmbH-Anteile nach Hagemeier eher fraglich sei. Das „Verschleiern der geschäftlichen Verhältnisse“ gem. $\$ 283$ I Nr. 8 Alt. 2 StGB wiederum sei nach dem BGH mit Blick auf die Gläubigerinteressen weit auszulegen; es genügten alle unklaren Angaben oder irreführenden Verhaltensweisen bzgl. unternehmensbezogener Umstände, was man hier annehmen könne. Der BGH deutet allerdings zugleich an, dass eine Strafbarkeit möglicherweise auszuschließen sei, wenn die Zahlungseinstellung (als objektive Bedingung der Strafbarkeit gem. $\$ 283$ VI StGB) bereits vor der Bankrotthandlung erfolgt sei, wie dies bei $\$ 283$ I Nr. 7 b StGB angenommen werde. Eine endgültige Entscheidung über diese Fragen traf der BGH allerdings nicht, da die entsprechenden Tatvorwürfe (neben einer erfolgten Verurteilung wegen Betrugs) gem. $\$ 154$ II StPO eingestellt worden waren. Die Erwägung einer Straflosigkeit aufgrund der bereits zuvor eingetretenen objektiven Strafbarkeitsbedingung wurde von Hagemeier kritisiert: es handele sich um eine Beschränkung der Strafbarkeit, für die insgesamt keine Begründung ersichtlich sei und die daher auch nicht auf $\$ 283$ I Nr. 8 StGB übertragen werden sollte.

Abschließend besprach Dr. Nina Nestler (Würzburg) eine Entscheidung des 3. Strafsenats des BGH (NStZ 2009, 437) die als „Abschied von der Interessentheorie“ bezeichnet wurde. Im konkreten Fall ging es um zwei Kommanditisten einer GmbH \& Co KG, die hoch verschuldet war und gegen die letztlich auf Betreiben der Banken das Insolvenzverfahren eröffnet wurde. Die beiden Kommanditisten wurden wegen Beihilfe zum Bankrott verurteilt; der BGH hob das Urteil auf und gab zugleich eine Art „Marschroute“ für das neue Verfahren. Im Kern ging es um die Frage der Anwendung von $\mathbb{\$} 14$ StGB im Rahmen des Bankrotttatbestandes, die für die Begründung der Strafbarkeit nötig ist. Bislang war dafür vom BGH ein Handeln (auch) im Interesse der Gesellschaft gefordert worden, insbesondere, um eine Abgrenzung zur gegen die Gesellschaft gerichteten strafbaren Untreue ( $\mathbb{2} 266 \mathrm{StGB})$ zu ermöglichen. Dem wurden allerdings schon länger gewichtige Argumente entgegen gehalten, u. a. Strafbarkeitslücken im Bereich von Verstößen gegen Buchführungs- und Bilanzierungspflichten, da hier ein Handeln im Interesse der Gesellschaft kaum vorstellbar sei. Auch könne sich unter Zugrundelegung der Interessentheorie der Geschäftsführer einer Ein-Mann-GmbH im Unterschied zum Einzelkaufmann bei rein eigennützigen, gegen das Gesellschaftsvermögen gerichteten Handlungen nicht nach $\mathbb{2 8 3}$ StGB strafbar machen. In einem obiter dictum äußerte nun der 3. Strafsenat in der 
oben erwähnten Entscheidung, die Interessentheorie im Rahmen von $\ 14$ StGB aus den genannten Argumenten nicht mehr anwenden zu wollen. Das bringt eine gewisse Klärung eines schon länger andauernden Meinungsstreits mit sich, ist allerdings, worauf Nestler zu Recht hinwies, mit einigen Folgefragen verbunden. Zum einen handele es sich nur um ein obiter dictum eines Senates, so dass abweichende Standpunkte anderer Senate weiterhin möglich blieben. Weiterhin sei nicht ganz klar, anhand welcher Kriterien in Zukunft innerhalb der Prüfung von $\mathbb{\$} 14$ StGB die Abgrenzung zu $\$ 266 \mathrm{StGB}$ erfolgen solle. Denkbar sei etwa eine objektiv-funktionale Betrachtung, bei der ein Handeln mit objektivem und eindeutigem Bezug zum Vertretungsverhältnis erforderlich sei oder ein Zurechnungsmodell im Sinne von Radtke, bei der das Handeln als „Geschäft des Vertretenen“ erscheinen müsse. Immerhin gibt der BGH Hinweise auf die von ihm präferierten Maßstäbe, wenn er fordert, dass der Täter „im Geschäftskreis des Vertretenen“ tätig werden müsse. Fraglich sei auch, ob die Entscheidung in Zukunft Auswirkungen auf die Reichweite des Untreuetatbestandes haben werde; so sei bisher ein den Tatbestand des $\$ 266$ StGB ausschließendes Einverständnis der Gesellschafter vom BGH abgelehnt worden, wenn dabei in einer bereits existenten Krise Interessen der Gläubiger beeinträchtigt wurden. Das Bedürfnis nach einer solchen (ohnehin zweifelhaften) extensiven Handhabung des Untreuetatbestandes könnte aufgrund der nun deutlich erweiterten Möglichkeit der Strafbarkeit gem. $\$ 283$ StGB entfallen sein.

Die Ergebnisse der Abschlussbesprechung wurden eingangs schon referiert. Man kann gespannt sein, wie sich die aus der Taufe gehobene Tagungsreihe entwickeln wird - ein verheißungsvoller Auftakt war die Kölner Veranstaltung allemal. 